${ }^{2}$ Gordon, T, and Kannel, W B, fournal of the American Medical Association, $1971,215,1617$

${ }^{3}$ Armstrong, A, et al, British Heart fournal, 1972, 34, 67.

4 McNeilly, R H, and Pemberton, J, British Medical fournal, 1968, 3, 139.

5 Dewar, H A, Fournal of the Royal College of Physicians, 1975, 9, 173.

6 White, N M, et al, British Medical fournal, 1973, 3, 618

7 Report of Joint Working Party, Fournal of the Royal College of Physicians, $1975,10,5$.

${ }^{8}$ Pantridge, J F, Geddes, J S, and Webb, S W, The Acute Coronary Attack. Tunbridge Wells, Pitman Medical, 1975.

9 Dikshit, K, et al, New England Fournal of Medicine, 1973, 288, 1087.

10 Shell, W E, and Sobel, B E, New England fournal of Medicine, 1974, 291, 481.

11 Das, G, Talmers, F N, and Weissler, A M, American fournal of Cardiology, $1975,36,281$.

\section{Whither scabies?}

Scabies occurs in epidemics, and, though it is still seen fairly often in Britain, there is reason to hope that the condition may soon become much less common. At the start of the second world war the mode of transmission of scabies was not fully understood, and in 1941 there were, according to Mellanby, ${ }^{1}$ over one million people in Britain with scabies. Mellanby's war-time experimental work on scabies transmission in volunteer conscientious objectors showed that scabies is not strictly a venereal disease and that a brief sexual encounter is less likely to transmit the infection than a more prolonged platonic sharing of a bed. Furthermore, his work showed that not only did regular bathing have little protective value but also that infrequent laundering of clothing and bedding did not increase the rate of transmission.

These facts suggested that the epidemic of scabies had less to do with wartime living conditions than had previously been believed. The disease had admittedly also been a scourge during the first world war, but a study of the children treated each year at a London cleansing centre had shown that the incidence of scabies had remained unchanged until 1920, when it had declined rapidly. ${ }^{2}$ Scabies was uncommon during the next 10 years, but it began to be seen more frequently in 1930, and the incidence rose precipitously again in 1937, well before the second world war started. The epidemic continued for the next 12 years, but the disease declined sharply in $1949^{3}$ and maintained a relatively low profile until 1964, when its incidence began to rise once more. ${ }^{4}$

Shrank and Alexander ${ }^{4}$ argued in 1967 that increased sexual promiscuity was unlikely to explain that latest increase, since there had been no concomitant increase in gonorrhoea or syphilis during the period of their study. Increased resistance of the mite to treatment was also excluded as a possibility on the grounds that no cases failed to respond to correct treatment. They also pointed out that each of the recent epidemics has lasted 10-15 years, with a similar quiescent period between epidemics. The highest incidence of scabies occurs in the age range from 15 to 30 years, so the facts are consistent with the theory that the "herd immunity" of the population gradually increases during an epidemic, and that afterwards about 15 years have to elapse for the partially immune population to be replaced by non-immune individuals.

Indeed, the concept of partial immunity to scabies was introduced by Mellanby, ${ }^{5}$ who showed that it is more difficult to induce a second attack of scabies and that during an established second attack fewer mites are found. The importance of immune responses in scabies is also suggested by the observation that the disease can be reactivated by methotrexate. ${ }^{6}$ A particularly severe variant called Norwegian scabies has been reported in patients with a specific anergy ${ }^{7}$ and in patients receiving immunosuppressive drugs. ${ }^{8}$ Some supportive laboratory evidence has come from a recent study showing that patients with scabies tend to have a low serum IgA concentration; if the serum values correlated with low IgA concentrations in the skin secretions, this might predispose to scabies infection. ${ }^{9}$ The possibility that the low IgA concentration was the result rather than the cause of the infection was not excluded, but the decrease was noted in early as well as late cases, and skin diseases are more usually associated with an increase in serum IgA. ${ }^{10}$ The relation between the serum IgA levels and the herd immunity to scabies remains to be elucidated.

If scabies runs true to form the present mild epidemic, which started in 1964, should be over by 1979 . Unfortunately, data from other countries show that the 30 -year cycle is by no means constant, ${ }^{11}$ and it may well be that scabies has not been common enough in Britain in recent years for herd immunity to have developed.

${ }^{1}$ Mellanby, K, Human Guinea-Pigs. London, Merlin Press, new edn, 1973.

2 Gray, A M H, British fournal of Dermatology and Syphilis, 1941, 53, 148.

${ }^{3}$ Epstein, E, Archives of Dermatology, 1955, 71, 192.

${ }^{4}$ Shrank, A B, and Alexander, Suzanne L, British Medical Fournal, 1967, 1,669 .

${ }^{5}$ Mellanby, K, Parasitology, 1944, 35, 197.

${ }^{6}$ Burrows, D, Bridges, J M, and Morris, T C M, British fournal of Dermatology, 1975, 93, 219.

7 Burks, J W Jr, Jung, R, and George, W M, Archives of Dermatology, 1956, 74, 131

${ }^{8}$ Paterson, W D, Allen, B R, and Beveridge, G W, British Medical fournal, 1973, 4, 211.

${ }^{9}$ Hancock, B W, and Ward, M, Fcurnal of Investigative Dermatology, 1974, 63,482 .

10 Lai, A, Fat, R F M, and van Furth, R, Clinical and Experimental Immunology, 1974, 17, 129.

11 Orkin, M, Fournal of the American Medical Association, 1971, 217, 593.

\section{Vaginal candidosis}

Symptoms resulting from infections of the vagina with yeasts are a growing problem among patients attending general practitioners, gynaecologists, genitourinary physicians, and venereologists. The usual cause is the fungus Candida albicans, but in a minority of cases symptoms may be due to Torulopsis glabrata. ${ }^{2}$ Candida is now the most common infectious agent found in women attending clinics for sexually transmitted diseases. ${ }^{13}$ For example, ${ }^{4}$ in 1973 in England and Wales 27545 cases were diagnosed in women at the clinics, an increase of $25 \%$ over 1971 ; the corresponding figure for men was 5058 cases, an increase of $68 \%$.

Whether or not clinical disease with symptoms results from infection depends on host factors, but little is known about the virulence of different strains of fungi. Factors such as glycosuria, diabetes, obesity, pregnancy, and the recent ingestion of antibiotics or steroids and other immunosuppressant agents have been known for some time to favour the growth of fungi in the vagina. Even so, the role of oral contraceptive pills is more controversial, particularly since the introduction of those with a lower oestrogen content.

The diagnosis can be established easily by identification of the mycelium and yeast spores on Gram-stained slides of specimens from the vaginal wall and cultures using a maltosepeptone agar such as Sabouraud's medium. One of the more difficult problems is to assess the meaning of asymptomatic candidosis in women, for the condition is quite frequently 
associated with other genital infections such as gonorrhoea or trichomoniasis.

In the absence of a safe and effective systemic antifugal agent treatment depends on the use of locally acting preparations. The principle is to apply the preparation to all the affected mucosal and skin areas for long enough to eliminate all the fungus and spores and at the same time to take measures to prevent reinfection by a sexual partner or from the patient's own gastrointestinal tract or elsewhere. Any predisposing factor should be eliminated if possible.

For years now the antifungal antibiotic, nystatin, has been the mainstay of treatment, used as vaginal pessaries, ointment, or cream. Oral tablets are available for gastrointestinal infections. Many other antifungal agents are incorporated into pessaries or ointments. Recently it has been suggested that better immediate and long-term results are obtained with ointments, ${ }^{5}$ though many patients complain that they are messy to use and difficult to apply even with an applicator. There is no evidence that the fungus develops any resistance to antifungal agents (with the one exception of amphotericin), and it is extremely difficult to assess whether any of the locally acting antifungal preparations is more effective than others; relapses are frequent and the treatment is far from satisfactory. In the long term a major advance in the treatment of candidosis will come only from the discovery of a safe and effective systemic agent, just as the treatment of trichomoniasis was revolutionised by the oral preparation metronidazole.

1 Oriel, J D, et al, British Medical fournal, 1972, 4, 761.

${ }^{2}$ Hurley, R, et al, fournal of Obstetrics and Gynaecology of the British Commonwealth, 1973, 80, 252.

${ }^{3}$ Catterall, R D, British fournal of Venereal Diseases, 1971, 47, 45.

${ }^{4}$ Department of Health and Social Security, Annual Report of the Chief Medical Officer for the Year 1974. On the State of the Public Health. London, HMSO, 1975.

${ }^{5}$ Morese, K. N, New York State Fournal of Medicine, 1975, 75, 1443.

\section{Rib pain}

Pain in the chest is a source of anxiety to both patient and doctor so long as its cause remains uncertain. Probably the most common cause is trauma to the rib cage from sporting activities, accidents, falls, or assault. Careful history, examination, and investigation will confirm the diagnosis but not always the severity of the injury- $x$-ray films often show only about half the rib fractures that actually exist, given what is found at necropsy. ${ }^{1}$ Complications may include haemothorax, pneumothorax, or the paradoxical movement of a "stove-in" chest, with respiratory embarrassment. Less commonly, where the uppermost ribs are fractured, there may also be a fracture of the clavicle and damage to the brachial plexus or the subclavian artery. Blunt trauma to the anterior chest wall may result in a fractured sternum and may cause mediastinal complications such as a tear in the arch of the aorta or cardiac contusion or tamponade. The first warning of these complications may be widening of the mediastinal shadow in the $x$-ray film, dysrhythmias or ischaemic changes on the ECG, or changes in central venous pressure respectively. A flexion fracture of the midthoracic spine may be seen in association with a fracture of the sternum and should therefore be considered in asking for specific radiological investigations.

The treatment of pain from simple rib fractures is oftengiven less consideration than the patient would like. Treatment should match individual circumstances. Strapping (applied in expiration) is usually best reserved for the young and active, whose lungs can accommodate the immobilisation. A simple test of the likely value of strapping is the relief given by the patient's own manual support of the injured area. In elderly patients restriction of respiratory movements may precipitate stasis and infection, so that strapping is contraindicated and analgesics are the treatment of choice. Valuable but less often employed is infiltration of the fracture site or block of the intercostal nerves by injection of a local anaesthetic.

$\mathrm{Rib}$ pain from obvious trauma causes little problem in diagnosis, but other mechanical causes of rib pain may be more puzzling. The cough fracture is well recognised, but less familiar is the pain caused by increased mobility of the anterior ends of the lower ribs. Described variously as the rib-tip syndrome, ${ }^{2}$ slipping rib, ${ }^{3}$ clicking rib, ${ }^{4}$ or nerve nipping at the intercostal margin, ${ }^{5}$ this condition may cause severe pain. It may be associated with a history of trauma and is usually described as a sharp pain related to movement or occasionally as a burning pain; it may be localised at the anterior end of the ribs or may radiate to the back.

$\mathrm{McBeath}$ and Keene recently investigated ${ }^{2}$ the anatomy of the anterior ends of the ribs and found that the eighth, ninth, and tenth ribs are attached anteriorly only to each other and by loose fibrous tissue. Trauma may make one of these ribs more mobile, and its movement may be palpable to the examining fingers and may result in direct pressure on the intercostal nerve. Radiological investigation will not disclose the condition, but manual pressure should reproduce the pain. The diagnosis of rib-tip syndrome should therefore be made on the history and findings at examination. Once it is confirmed, reassurance may be sufficient for the over-anxious, while infiltration with local anaesthetic and steroid preparations may relieve troublesome symptoms. In those with severe or recurring pain surgical excision of the rib tip offers a permanent cure.

Tietze's disease is another less common cause of rib pain, characterised by a painful non-suppurative swelling of the second or third costal cartilage: again the chest $x$-ray film is normal. Treatment may vary from reassurance to the application of local heat or the local injection of hydrocortisone.

Radiological examination of a painful rib swelling may occasionally show a benign or malignant tumour. Benign tumours, often symptomless, rarely cause pathological fractures; they include chondromas, multiple exostoses, solitary myeloma, and fibrous dysplasia, and in the first removal is usually advised because there is a risk of malignant change. Primary malignant tumours are rare, and the most likely is a chondrosarcoma. Typically, metastases in the rib are associated with a primary lesion in the lung or breast, though other primary sites may be concerned. Such secondary deposits are almost invariably painful and tender.

Pain in the rib cage may also be caused by a neurofibroma affecting the intercostal nerve or a ganglioneuroma arising from the sympathetic chain. Such tumours may cause mechanical and neurological symptoms from pressure, but more often than not they are symptomless and single.

Finally the rib cage may encompass a unilateral or bilateral extra member, the cervical rib, a congenital fibrous or bony extension from the seventh cervical vertebra. Cervical ribs rarely cause local symptoms: more common are neurological features in the arm varying from pain and paraesthesiae from pressure on the brachial plexus to the less frequent vasomotor or vascular symptoms. In both instances detailed examination, including the effect of shoulder movement and mapping the distribution of neurological changes, will narrow the diagnostic 